\title{
Methotrexate for Inflammatory Bowel Diseases - New Developments
}

\author{
Hans H. Herfarth \\ Division of Gastroenterology and Hepatology, Department of Medicine, University of North Carolina, \\ Chapel Hill, N.C., USA
}

\section{Key Words}

Crohn's disease - Ulcerative colitis - Methotrexate $\cdot$ Anti-TNF therapy $\cdot$ Infliximab · Azathioprine

\begin{abstract}
Methotrexate (MTX) is an established therapy for patients with steroid-dependent Crohn's disease (CD). MTX is also frequently used in combination with anti-TNF agents to suppress anti-drug antibody formation. It has been suggested in the past that MTX lacks any clinical effectiveness in patients with ulcerative colitis (UC); however, newer data at least partially contradict this assumption. The following review will discuss recent data for the use of MTX in CD, UC and in combination with anti-TNF agents.

(c) 2016 S. Karger AG, Basel
\end{abstract}

\section{Introduction}

In 1989, Kozarek et al. [1] were the first to report a beneficial effect of intramuscular methotrexate (MTX) therapy in 21 patients with refractory Crohn's disease (CD) or ulcerative colitis (UC). It took an additional 6 years to confirm the clinical efficacy of MTX as induction regimen and yet another 13 years as maintenance therapy in patients with CD. Two landmark trials of the North American Crohn's Study Group Investigators published in 1995 and 2000 established that 25 mg MTX giv- en intramuscularly once weekly for induction and $15 \mathrm{mg}$ MTX given intramuscularly once weekly for maintenance was more effective than placebo in improving clinical symptoms and reducing requirements for prednisone [2, $3]$. Still in the last 20 years, MTX has rarely been used for the treatment of CD despite robust clinical evidence as illustrated by low prescription rates in large insurer databases [4-7]. This is astonishing considering that MTX is a generic medication and one of the very few reasonably priced drugs with a proven clinical value in the treatment of steroid-dependent CD. Possible reasons for the lack of success in incorporating MTX in the routine CD treatment algorithm might be the need for subcutaneous (s.c.) or intramuscular injections, the relative high incidence of nausea (around 20\%) if the patient is not treated with concurrent anti-nausea medications and the lack of additional assuring prospective studies in CD. However, more recently, MTX therapy is becoming more in vogue in pediatric patients and is also being investigated as a potential therapeutic approach in patients with UC [810].

\section{MTX Therapy in Pediatric and Adult CD Patients - New Data}

Since the first reports of an increased rate of hepatosplenic lymphoma (HDSTCL) in the setting of a combination therapy of a thiopurine with an anti-TNF agent in

\section{KARGER}

E-Mail karger@karger.com

www.karger.com/ddi
(C) 2016 S. Karger AG, Base

0257-2753/16/0342-0140\$39.50/0
Hans $\mathrm{H}$. Herfarth, MD, $\mathrm{PhD}$

103 Mason Farm Road

CB 7080

Chapel Hill, NC 27599 (USA)

E-Mail hherf@med.unc.edu 
young males in 2006, many pediatric centers have reduced the utilization of azathioprine (AZA) or 6-mercaptopurine (6-MP) in pediatric patients $[11,12]$. According to a recent multi-center study, since 2006 , a significant trend occurred toward the preferred use of MTX as a firstline immunomodulator (fig. 1) [12].

MTX monotherapy in pediatric CD patients has been shown to be successful in inducing steroid-free remission and subsequently maintaining this remission in approximately $65 \%$ of the patients over the next 5 years $[12,13]$. Consequently, it is highly likely that a considerable number of pediatric patients will transition to adult providers in the next years while being in remission on long-term MTX therapy. In this scenario, questions of long-term toxicity, specifically regarding liver toxicity, are likely to arise. Currently, liver biopsies are only recommended in the setting of reproducible elevations of liver function tests; however, liver fibrosis or cirrhosis may also develop in the absence of abnormal liver values $[10,14]$. Thus, a non-invasive method to monitor patients on long-term MTX therapy would be desirable. Possibilities to monitor for liver toxicity in a non-invasive manner might be the use of transient elastography (Fibroscan) [15]. However, currently, no longitudinal studies evaluating this technology are available [10].

Whereas the usefulness of MTX in adults in the first year has been analyzed in several studies, the availability continuous efficacy data beyond 1 year duration of therapy have been scarce [4]. Hausmann et al. [16] performed a meta-analysis of 4 studies, including $267 \mathrm{CD}$ patients with long-term follow-up [16-20]. In this analysis, it becomes apparent that the cumulative probability to maintain remission on MTX monotherapy decreases by approximately $30 \%$ over a 3 -year time period (fig. 2 ), which seems contradictory to the above reported results in pediatric patients. A similar decrease of slightly more than $40 \%$ of sustained clinical benefit from 63 to $47 \%$ and $20 \%$ in years 1,2 and 5 , respectively, after therapy initiation is reported in a multi-center analysis from the Netherlands [21]. Even higher loss of response rates are described in a smaller study from a single center in England with a cessation of clinical benefit in $70 \%$ of the patients over a 3 -year time period [22]. Multiple factors might influence the long-term effectiveness of MTX in CD including medication compliance, lower and ineffective maintenance doses of MTX or as yet undefined escape mechanisms of the immune system, which lead to re-occurrence of intestinal inflammation despite ongoing immunosuppressive therapy.

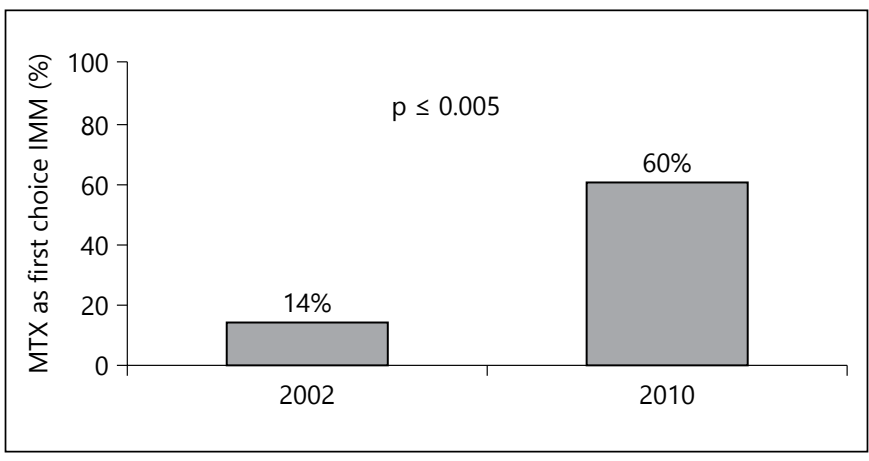

Fig. 1. Trends of MTX use by year. Shown is the fraction of MTX as first choice immunomodulator in a prospective pediatric inception cohort study in 2002 and by 2010 . There was no gender difference in this trend [12].

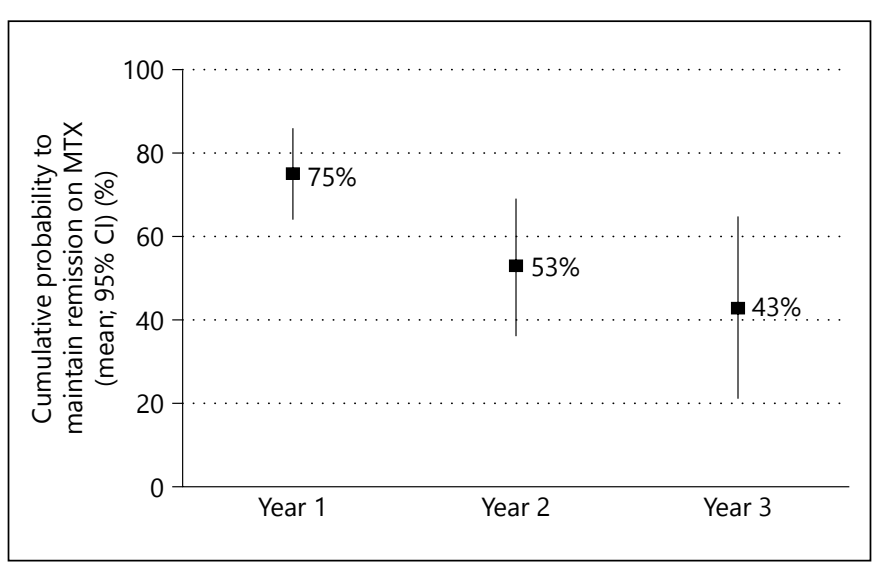

Fig. 2. Cumulative probability to maintain remission on MTX in CD over 3 years [16].

\section{New Data of MTX and Anti-TNF Combination Therapy}

AZA/6-MP and MTX appear to be equally effective in suppressing antibody formation and preserving higher infliximab (IFX) trough levels [23]. Subsequently, the Study of Biologic and Immunomodulator Naive Patients in CD (SONIC) study revealed a greater clinical effectiveness of a combination approach of IFX and AZA compared to IFX alone [24]. One major factor for the increased efficiency of a combination approach is thought to be a lower likelihood of anti-IFX antibody formation and higher IFX trough levels. However, the currently available data of a clinical advantage of combination therapy appear not to be as convincing for other anti-TNF 
agents than IFX. MTX and anti-TNF combination therapy seems to prolong the long-term efficacy of all FDAapproved anti-TNF agents in patients with rheumatoid arthritis [25]. Yet currently, there is a lack of any prospective or large retrospective analyses in patients with IBD describing an increase in the short- or long-term efficacies and durability of a combination therapy of AZA/6MP or MTX with one of the humanized anti-TNF antibodies adalimumab, certolizumab and golimumab [26]. Furthermore, the results of recently published study 'The Combination of Maintenance Methotrexate-Infliximab Trial (COMMIT)' did not help to further elucidate the important question of a mono versus a combination approach in the setting of biological therapy [27]. COMMIT revealed that the addition of MTX to IFX leads to a significant suppression of antibody formation to IFX and higher serum IFX trough levels compared to IFX monotherapy. In contrast to the SONIC, these serologic results did not correlate with an improvement in clinical outcome during the 50 weeks duration of the COMMIT. The reasons for the differences between COMMIT and SONIC can only be speculated upon, but several factors, which might have influenced the divergent outcomes in both trials, have been suggested [28]. These include different disease duration before inclusion in the trial in the SONIC versus COMMIT (2.2 vs. 9 years), different inclusion criteria in regard to previous exposure to immunosuppressive medication and concurrent steroid therapy and 'dual' therapy in SONIC (IFX + AZA) versus 'triple' therapy in the COMMIT (initial steroid taper + single steroid application before each IFX infusion). Also, in contrast to the SONIC findings, a recent study investigating the benefit of continuing AZA or 6-MP when starting anti-TNF agents (IFX or adalimumab) in the setting of step-up therapy suggested a higher risk of opportunistic infections but no clinical benefit of a combination therapy [29]. Data from a large pediatric registry recently revealed that in boys, the overall durability of IFX therapy with concomitant MTX for $\geq 6$ months after starting IFX was significantly better than with concomitant thiopurines [30]. At present, no firm conclusion with regard to the question 'which is the better combination therapy with IFX MTX or AZA/6-MP?' can be drawn [31]. Perhaps a recently funded pragmatic trial, which aims to compare the effectiveness of anti-TNF alone or in combination with oral MTX (http://www.pcori.org/researchresults/2015/anti-tnf-monotherapy-versus-combination-therapy-low-dose-methotrexate), can help to further clarify the role of MTX in its role as anti-TNF adjunctive therapy. Additionally, the role of AZA/6-MP or MTX in combination with the other approved antiTNF agents - adalimumab, certolizumab and golimumab - needs further exploration in clinical studies. Moreover, there remain open questions as to the dose and modality of MTX therapy: do lower doses (e.g. $15 \mathrm{mg}$ ) have the same clinical efficacy as the higher dose of MTX in combination therapy? In maintaining remission in patients with CD, $15 \mathrm{mg}$ MTX s.c. seems to be inferior to $25 \mathrm{mg}$, but in combination therapy this might be different. Do the application modalities have an influence on efficacy and durability of combination therapy (s.c. vs. oral)? MTX monotherapy is more effective if applied s.c. then orally, but again this might not have an impact in the setting of combination with anti-TNF [32-34]. A recent retrospective chart review at a tertiary care center in the US addressed both questions [35]. The study included 88 IBD patients $(74 \% \mathrm{CD}, 22 \% \mathrm{UC}, 4 \%$ indeterminate colitis) on anti-TNF therapy ( $49 \%$ on adalimumab, $40 \%$ on IFX and $11 \%$ on certolizumab), who were also treated with concomitant MTX therapy. The results revealed that patients on MTX doses of $>12.5 \mathrm{mg} /$ week had a higher likelihood to remain in clinical remission than those on lower doses and there was also a trend toward higher efficacy of parenteral versus oral MTX application. The results of the study are highly interesting but the overall number of included patients is small especially given the multiple analyses (3 different underlying disease conditions - CD, UC, indeterminate colitis; 3 different antiTNF regimen, different MTX dose and application regimen). Clearly more research is needed in this direction. However, the fact that doses of $12.5 \mathrm{mg}$ MTX per week might be too low to have any significant clinical efficacy is supported by yet another retrospective analysis of the durability of IFX therapy [36]. In this single center analysis including pediatric patients, MTX doses $<10 \mathrm{mg} /$ week were ineffective, since no difference in outcome was observed between children on IFX/MTX combination therapy compared to those on IFX monotherapy.

\section{MTX in UC - Is It Therapeutically Effective?}

A multi-center study directed by Oren et al. [37] investigated the efficacy of oral MTX at a dose of $12.5 \mathrm{mg}$ orally per week compared to placebo in 67 patients with at least moderately active UC in the early 1990s. The treatment duration was 9 months; 5 -aminosalicylates (5-ASA) and steroids were allowed to be continued during the study at the discretion of the treating physician and the primary outcome measures were the proportion of pa- 
Fig. 3. METEOR results at week 16: steroid-free clinical and endoscopic remission and steroid-free clinical remission only [39].

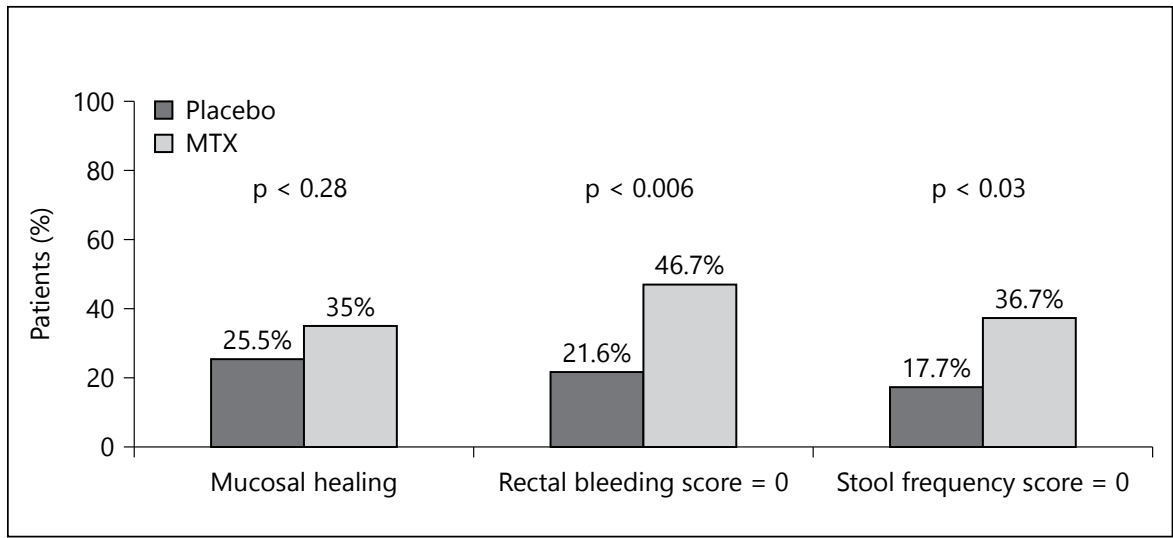

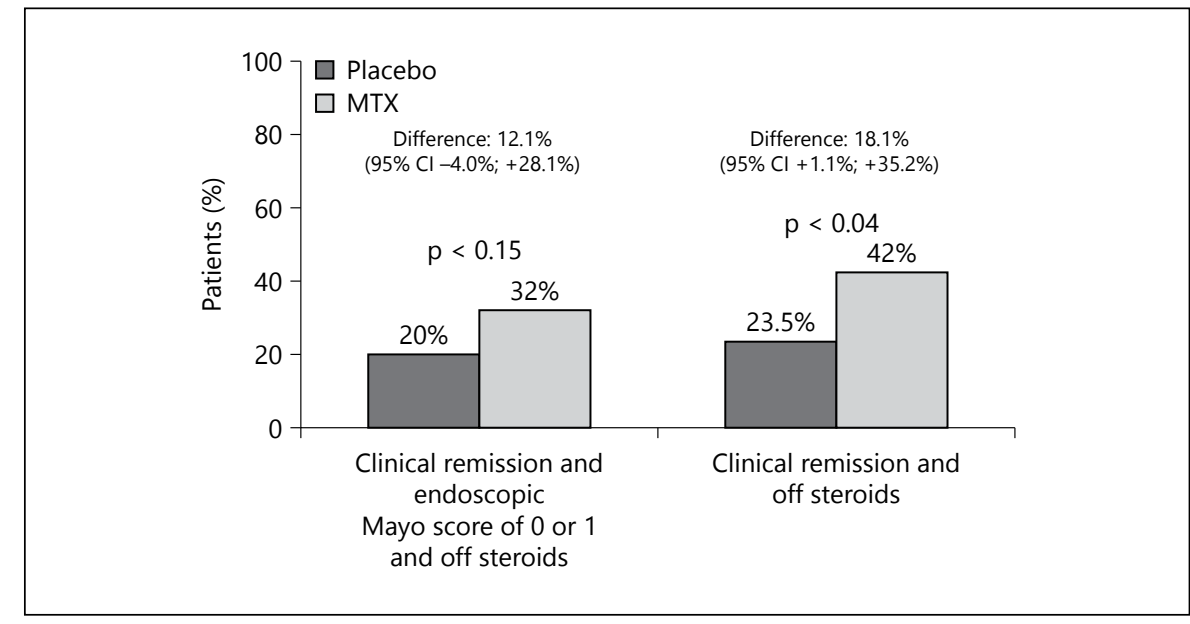

Fig. 4. METEOR endoscopic and clinical end points at week 16: endoscopic healing defined as Mayo endoscopic subscore $=0$ or 1 and patient reported outcomes of absence of rectal bleeding and normalization of bowel frequency [39]. tients entering first remission, time to reach that remission and maintenance of remission. There were no significant differences among the groups with regard to the primary outcomes, monthly steroid use, clinical Mayo scores or mucosal healing. Despite the positive results of 3 other very small prospective studies, which also had significant qualitative weaknesses, the results from Oren et al. [37] led to the conclusion that MTX is ineffective in the therapy of UC $[9,38]$. However, data from the recently presented METEOR (comparison of MTX vs. placebo in corticosteroid-dependent ulcerative colitis) trial suggest a significant clinical efficacy in inducing steroid-free remission [39]. The aim of this trial was the investigation of s.c. applied MTX $25 \mathrm{mg} /$ week as an induction regimen over 16 weeks. MTX was superior to placebo (42.0 vs. $23.5 \%, \mathrm{p}<0.04)$, when the investigators just analyzed the clinical efficacy excluding the sigmoidoscopy results (fig. 3). However, the primary end point was a combined end point of the clinical and endoscopic Mayo score with a total score $\leq 2$ and no item $>1$, complete steroid withdrawal with a forced steroid tapering regimen and no need for other immunosuppressants, anti-TNF or colectomy at week 16 . With the addition of the endoscopy results, the study missed the primary end point (fig. 3). Interestingly, the absence of rectal bleeding and normalization of stool frequency, both outcomes that are normally considered a surrogate marker of mucosal healing, were significantly better in patients on MTX than those on placebo (fig. 4) [40-42]. Overall, the study was most likely underpowered since the investigators had assumed that MTX would have a $45 \%$ probability of steroid-free remission, which is better than any currently available drugs for the treatment of UC. Other limitations of the METEOR design comprise the inclusion of patients with clinical and/or endoscopic inactive disease (but being steroid dependent) and the absence of central reading of the endos- 
Fig. 5. Trial design of the MERIT-UC study with open label induction period and placebo-controlled maintenance period after randomization at week 16 .

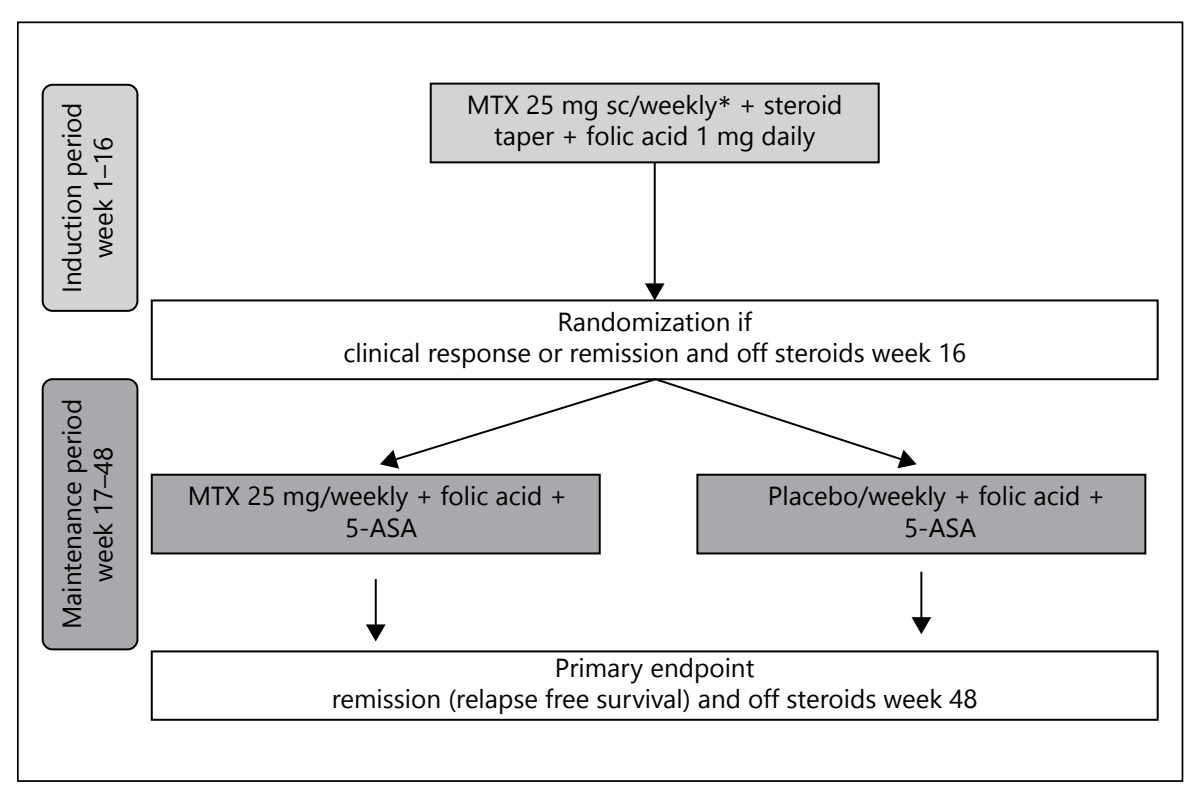

copy scores. To put the METEOR results into perspective, the randomized prospective study compared AZA with IFX monotherapy and IFX/AZA combination therapy (SUCCESS trial) had a similar end point as METEOR and demonstrated steroid-free remission for 24, 22 and 40\% of the patients, respectively [43]. Of note, the SUCCESS study population significantly differed from that of METEOR since only anti-TNF and AZA naïve patients or patients who had stopped AZA at least for 3 months before the study were included.

Currently a second study, which is sponsored by the National Institute of Health and performed by the Crohn's and Colitis Foundation of America - Clinical Research Alliance, is analyzing the efficacy of MTX in maintaining steroid-free remission and is currently ongoing (randomized, double blind, prospective trial investigating the efficacy of MTX in induction and maintenance of steroid free remission in ulcerative colitis (MEthotrexate Response in Treatment of UC - MERIT-UC); ClinicalTrials.gov, NCT01393405). This study follows a withdrawal design similar to the landmark CD MTX maintenance study by Feagan et al. [2]. Patients who had failed at least one previous UC therapy (5-ASA, AZA/6-MP, anti-TNF or vedolizumab and/or are steroid dependent) are treated with open label MTX $25 \mathrm{mg}$ s.c. per week with a concomitant steroid taper (fig. 5). The steroid taper has to be finished at week 12 . If the patients are responding or are in remission at week 16, they are randomized to placebo or continuation of MTX therapy for another 32 weeks. Interims results are available for the first 96 pa- tients who have completed the 16 weeks. Thirty percent of the patients on open label MTX were in clinical remission defined by a clinical Mayo score $\leq 2$, and $50 \%$ of all patients started on MTX were in clinical response defined as decrease in the clinical Mayo score of $\geq 2$ points and at least a $25 \%$ decrease from baseline Mayo score. The MERIT-UC trial is still ongoing and the final results of the placebo-controlled maintenance phase are expected by 2017 .

\section{Summary}

MTX is becoming the preferred immunosuppressive agent in the therapy of pediatric $\mathrm{CD}$ either as mono or in combination therapy with biologics. The long-term efficacy in pediatric and adult patients appears to be comparable to AZA/6-MP therapy. In combination therapy with anti-TNF agents, MTX has similar ability as AZA/6MP to suppress IFX antibody formation and to increase the IFX trough level. Current evidence suggests that MTX should be administered at least at a dose $\geq 12.5 \mathrm{mg}$ weekly to improve the efficacy of anti-TNF therapy; however, there is still some debate about the optimal dose and the mode of application. The merits of MTX in inducing and maintaining steroid-free remission in patients with active UC are still unresolved. The recently published results of the METEOR study missed the primary combined end point of steroid-free clinical and endoscopic remission. However, a significant clinical effect 
of MTX compared to placebo, which was not matched by a significant improvement of mucosal inflammation suggest that the study was most likely underpowered. Thus, METEOR ultimately failed to prove or refute the therapeutic effectiveness of MTX in UC. Hopefully, the results of MERIT-UC will be able to finally resolve the ongoing debate on the efficacy of MTX in patients with active UC.

\section{Acknowledgments}

This work is supported by National Institutes of Health grant 1U01-DK092239-01.

\section{Disclosure Statement}

No disclosures.

\section{References}

1 Kozarek RA, Patterson DJ, Gelfand MD, et al: Methotrexate induces clinical and histologic remission in patients with refractory inflammatory bowel disease. Ann Intern Med 1989; 110:353-356.

2 Feagan BG, Fedorak RN, Irvine EJ, et al: A comparison of methotrexate with placebo for the maintenance of remission in Crohn's disease. North American Crohn's Study Group Investigators. N Engl J Med 2000;342:16271632.

3 Feagan BG, Rochon J, Fedorak RN, et al: Methotrexate for the treatment of Crohn's disease. The North American Crohn's Study Group Investigators. N Engl J Med 1995;332: 292-297.

4 Patel V, Wang Y, MacDonald JK, et al: Methotrexate for maintenance of remission in Crohn's disease. Cochrane Database Syst Rev 2014;8:CD006884.

5 McDonald JW, Wang Y, Tsoulis DJ, et al: Methotrexate for induction of remission in refractory Crohn's disease. Cochrane Database Syst Rev 2014;8:CD003459.

6 Herfarth HH, Long MD, Isaacs KL: Methotrexate: underused and ignored? Dig Dis 2012;30(suppl 3):112-118.

7 Benchimol EI, Cook SF, Erichsen R, et al: International variation in medication prescription rates among elderly patients with inflammatory bowel disease. J Crohns Colitis 2013; 7:878-889.

8 Vaysse T, Carbonnel F: Methotrexate in IBD: the return of the prodigal son. J Crohns Colitis 2015;9:303-304.

9 Herfarth HH, Kappelman MD, Long MD, Isaacs KL: Efficacy of methotrexate in ulcerative colitis: failure or promise. Inflamm Bowel Dis 2010;16:1421-1430.

10 Herfarth HH, Kappelman MD, Long MD, et al: Use of methotrexate in the treatment of inflammatory bowel diseases. Inflamm Bowel Dis 2016;22:224-233.

11 Rosh JR, Gross T, Mamula P, et al: Hepatosplenic T-cell lymphoma in adolescents and young adults with Crohn's disease: a cautionary tale? Inflamm Bowel Dis 2007;13:1024-1030.

12 Sunseri W, Hyams JS, Lerer T, et al: Retrospective cohort study of methotrexate use in the treatment of pediatric Crohn's disease. Inflamm Bowel Dis 2014;20:1341-1345.
13 Uhlen S, Belbouab R, Narebski K, et al: Efficacy of methotrexate in pediatric Crohn's disease: a French multicenter study. Inflamm Bowel Dis 2006;12:1053-1057.

14 Dassopoulos T, Sultan S, Falck-Ytter YT, et al: American Gastroenterological Association Institute technical review on the use of thiopurines, methotrexate, and anti-TNF- $\alpha$ biologic drugs for the induction and maintenance of remission in inflammatory Crohn's disease. Gastroenterology 2013;145:14641478.e1-e5.

15 Kaffenberger BH, Kaffenberger JA, Wong H, et al: Magnetic resonance elastography and transient elastography as non-invasive analyses for liver fibrosis: can they obviate the need for liver biopsy in psoriasis patients treated with methotrexate? Int J Dermatol 2015;54: 752-756.

16 Hausmann J, Zabel K, Herrmann E, et al: Methotrexate for maintenance of remission in chronic active Crohn's disease: long-term single-center experience and meta-analysis of observational studies. Inflamm Bowel Dis 2010;16:1195-1202.

17 Domenech E, Manosa M, Navarro M, et al: Long-term methotrexate for Crohn's disease: safety and efficacy in clinical practice. J Clin Gastroenterol 2008;42:395-399.

18 Lemann M, Zenjari T, Bouhnik Y, et al: Methotrexate in Crohn's disease: long-term efficacy and toxicity. Am J Gastroenterol 2000;95: 1730-1734.

19 Chong RY, Hanauer SB, Cohen RD: Efficacy of parenteral methotrexate in refractory Crohn's disease. Aliment Pharmacol Ther 2001; 15:35-44.

20 Charpignon C, Beau P: Methotrexate as single therapy in Crohn's disease: is its long-term efficacy limited? Gastroenterol Clin Biol 2008; 32:153-157.

21 Seinen ML, Ponsioen CY, de Boer NK, et al: Sustained clinical benefit and tolerability of methotrexate monotherapy after thiopurine therapy in patients with Crohn's disease. Clin Gastroenterol Hepatol 2013;11:667-672.

22 Suares NC, Hamlin PJ, Greer DP, et al: Efficacy and tolerability of methotrexate therapy for refractory Crohn's disease: a large singlecentre experience. Aliment Pharmacol Ther 2012;35:284-291.
23 Vermeire S, Noman M, Van Assche G, et al: Effectiveness of concomitant immunosuppressive therapy in suppressing the formation of antibodies to infliximab in Crohn's disease. Gut 2007;56:1226-1231.

24 Colombel JF, Sandborn WJ, Reinisch W, et al: Infliximab, azathioprine, or combination therapy for Crohn's disease. N Engl J Med 2010;362:1383-1395.

25 Zhang J, Xie F, Delzell E, et al: Impact of biologic agents with and without concomitant methotrexate and at reduced doses in older rheumatoid arthritis patients. Arthritis Care Res (Hoboken) 2015;67:624-632.

26 Dulai PS, Siegel CA, Colombel JF, et al: Systematic review: monotherapy with antitumour necrosis factor $\alpha$ agents versus combination therapy with an immunosuppressive for IBD. Gut 2014;63:1843-1853.

27 Feagan BG, McDonald JW, Panaccione R, et al: Methotrexate in combination with infliximab is no more effective than infliximab alone in patients with Crohn's disease. Gastroenterology 2014;146:681-688.e1.

28 Narula N, Peyrin-Biroulet L, Colombel JF: Combination therapy with methotrexate in inflammatory bowel disease: time to COMMIT? Gastroenterology 2014;146:608-611.

29 Osterman MT, Haynes K, Delzell E, et al: Effectiveness and safety of immunomodulators with anti-tumor necrosis factor therapy in Crohn's disease. Clin Gastroenterol Hepatol 2015;13:1293-1301.e5; quiz e70, e72.

30 Grossi V, Lerer T, Griffiths A, et al: Concomitant use of immunomodulators affects the durability of infliximab therapy in children with Crohn's disease. Clin Gastroenterol Hepatol 2015;13:1748-1756.

31 Aloi M, Cucchiara S: Predicting the durability of biological therapy in pediatric Crohn's disease: do the immunomodulators matter? Clin Gastroenterol Hepatol 2015;13:1757-1759.

32 Hazlewood GS, Thorne JC, Pope JE, et al: The comparative effectiveness of oral versus subcutaneous methotrexate for the treatment of early rheumatoid arthritis. Ann Rheum Dis 2015;pii:annrheumdis-2014-206504.

33 Turner D, Doveh E, Cohen A, et al: Efficacy of oral methotrexate in paediatric Crohn's disease: a multicentre propensity score study. Gut 2015;64:1898-1904. 
34 Schiff MH, Jaffe JS, Freundlich B: Head-tohead, randomised, crossover study of oral versus subcutaneous methotrexate- in patients with rheumatoid arthritis: drug-exposure limitations of oral methotrexate at doses $\geq 15 \mathrm{mg}$ may be overcome with subcutaneous administration. Ann Rheum Dis 2014;73: 1549-1551.

35 Colman RJ, Rubin DT: Optimal doses of methotrexate combined with anti-TNF therapy to maintain clinical remission in inflammatory bowel disease. J Crohns Colitis 2015; 9:312-317.

36 Vahabnezhad E, Rabizadeh S, Dubinsky MC: A 10-year, single tertiary care center experience on the durability of infliximab in pediatric inflammatory bowel disease. Inflamm Bowel Dis 2014;20:606-613.
37 Oren R, Arber N, Odes S, et al: Methotrexate in chronic active ulcerative colitis: a doubleblind, randomized, Israeli multicenter trial. Gastroenterology 1996;110:1416-1421.

38 Wang Y, MacDonald JK, Vandermeer B, et al: Methotrexate for maintenance of remission in ulcerative colitis. Cochrane Database Syst Rev 2015;8:CD007560.

39 Carbonnel F, Colombel JF, Filippi J, et al: 745 methotrexate for corticosteroid-dependent ulcerative colitis: results of a placebo randomized controlled trial. Gastroenterology 2015; 148:S1-S140.
40 Higgins PD, Schwartz M, Mapili J, et al: Is endoscopy necessary for the measurement of disease activity in ulcerative colitis? Am J Gastroenterol 2005;100:355-361.

41 Lewis JD, Chuai S, Nessel L, et al: Use of the noninvasive components of the Mayo score to assess clinical response in ulcerative colitis. Inflamm Bowel Dis 2008;14:1660-1666.

42 Jharap B, Sandborn WJ, Reinisch W, et al: Randomised clinical study: discrepancies between patient-reported outcomes and endoscopic appearance in moderate to severe ulcerative colitis. Aliment Pharmacol Ther 2015;42:1082-1092.

43 Panaccione R, Ghosh S, Middleton S, et al: Combination therapy with infliximab and azathioprine is superior to monotherapy with either agent in ulcerative colitis. Gastroenterology 2014;146:392-400.e3. 